\title{
VARIATIONALLY-BASED EFFECTIVE DYNAMIC THICKNESS FOR LAMINATED GLASS BEAMS
}

\author{
Jaroslav Schmidt*, Alena Zemanová, Tomáš Janda, Jan Zeman, \\ Michal ŠEJNOHA \\ Czech Technical University in Prague, Faculty of Civil Engineering, Thákurova 7, 16629 Prague 6, Czech \\ Republic \\ * corresponding author: jarasit@gmail.com
}

\begin{abstract}
Laminated glass, consisting of glass layers connected with transparent foils, has found its applications in civil, automotive, or marine engineering. Due to a high contrast in layer properties, mechanical response of laminated glass structures cannot be predicted using classical laminate theories. On the other hand, engineering applications demand easy-to-use formulas of acceptable accuracy. This contribution addresses such simplified models for free vibrations of laminated glass beams, with the goal to determine their natural frequencies and modal damping properties. Our strategy is to approximate the complex behavior of a laminated structure with that of an equivalent monolithic beam. Its effective thickness is determined by the variational method proposed by Galuppi and Royer-Carfagni for static problems, which we extended for modal analysis. We show that this new approach overcomes inaccuracies of the currently used dynamic effective thickness model by López-Aenlle and Pelayo.
\end{abstract}

KeYwords: Laminated glass, dynamic effective thickness, modal analysis.

\section{Motivation}

In the past, glass was seen as an infill material for transparent areas with no load-bearing function in a structural system. The invention of laminated glass became one of the milestones that allowed glass to attain a genuine structural role [1]. Starting from windscreens in the car industry, its use has expanded into other fields of industry, including civil engineering. Today, layered glass is used, for example, for floor and roof systems, columns, staircases etc.

Laminated glass is a layered composite material consisting of several layers of glass and polymer. Apart from its primary function in enhancing the post-peak load-bearing capacity, the polymer interlayer also provides damping of vibrations [2]. Therefore, it can also reduce the possible noise and vibration problems, which can occur in structures under dynamic loading.

On the other hand, the nature of the polymer response is strongly frequency-dependent and also temperature-sensitive, e.g., [3], [4], or [5]. Its behavior is often described by a complex-valued dynamic shear modulus, see Figure 1 This complex-valued formulation allows us to transmit the information about the resonance behavior and damping of the structural element in a single complex number. For these reasons, the eigenvalue problem which describes the free vibrations of laminated glass becomes complex and nonlinear; see Section 2.1 for more details. To the best of our knowledge, such type of problems cannot be currently addressed with standard finite element systems used in civil engineering.

As engineering practice demands easy-to-use approaches of acceptable accuracy, this contribution addresses simplified models for free vibrations of laminated glass beams, with the goal to determine their natural frequencies and modal damping properties. Our focus is on the effective thickness approaches one group of the simplified methods popular in the design of laminated glass structures, e.g., [6], [7, 8]. In particular, we demonstrate on selected examples the (in)accuracy of the state-of-the-art effective thickness approaches from literature, and we discuss our proposal how to increase their accuracy by employing principles of variationally-based effective thickness methods.

The structure of the paper is as follows. In Section 2 we briefly describe all approaches used in this study. The examples are introduced and the results of our comparison are discussed in Section 3 . Finally, the main findings of our study are summarized in Section 4

\section{Methods}

Three methods for vibration analysis of laminated glass beams are used in this section. The first method is based on solving the nonlinear and complex eigenvalue problem using a finite element discretization and the Newton method. This method provides us with a reference solution to assess the accuracy of effective thickness approaches. The second method is the dynamic effective thickness method (DET) proposed by López-Aenlle and Pelayo [6]. Finally, the last method is derived from the enhanced effective thickness approach (EET) by Galuppi and Royer-Carfagni for deflections under static loading [7], which we adjusted for modal analysis. 


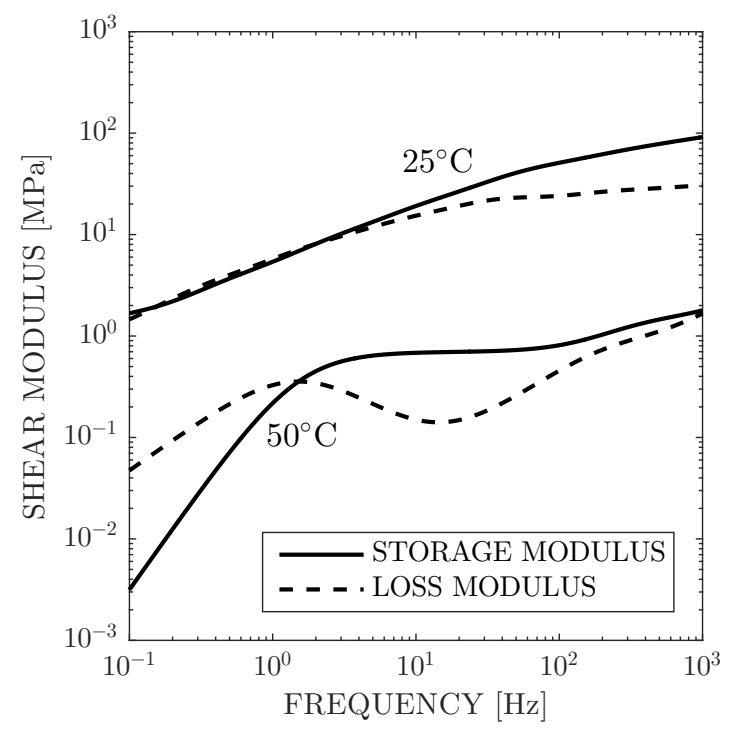

Figure 1. The frequency dependence of the real and imaginary part of the complex shear modulus for polyvinyl butyral (PVB) according to $[3]$.

For the sake of simplicity, we focus on the most common case of laminated glass configuration - a threelayered beam. However, the generalization of all methods for multi-layered beams can be also found in the literature [9]. In our analysis, we consider a threelayered beam with the same material parameters for both glass layers and with constant properties along the length according to Figure 2 Then, the crosssection areas $A_{i}$ and the second moments of area $I_{i}$ are defined by the standard relations

$$
I_{i}=\frac{1}{12} b h_{i}^{3}, \quad A_{i}=b h_{i}, \quad i=\{1,2,3\},
$$

where $b$ and $h_{i}$ stand for the width and the thicknesses of the layers; see Figure 2 .

\subsection{REFERENCE METHOD (RM)}

Our reference method is based on the finite element method (FEM) together with the Newton method due to the nonlinearity of the solved eigenvalue problem. The system of equations of natural vibrations is written in the FEM matrix form as follows

$$
\left(\boldsymbol{K}(\omega)-\omega^{2} \boldsymbol{M}\right) \boldsymbol{U}=\mathbf{0},
$$

where $\omega$ is the natural angular frequency, $\boldsymbol{U}$ is the corresponding vector of the mode shape, $\boldsymbol{K}(\omega)$ is the complex frequency-dependent stiffness matrix, and $\boldsymbol{M}$ is the real constant mass matrix. The eigenvalue problem described by Eq. (2) specifies the mode shape up to an arbitrary constant. Therefore, we use a regularization condition

$$
\boldsymbol{U}_{0}^{\top}\left(\boldsymbol{U}-\boldsymbol{U}_{0}\right)=0,
$$

in order to ensure the uniqueness of the solution. Here, $\boldsymbol{U}_{0}$ is the initial mode shape solving the real eigenvalue problem with the constant stiffness matrix accounting only for the initial shear modulus of the polymer interlayer.

For each initial eigen-pair $\omega_{0}$ and $\boldsymbol{U}_{0}$, we solve iteratively a linearized system of equations, derived by the Newton method [10] from Eq. (2) and Eq. (3), until the convergence is achieved. Then, the natural frequencies $f_{\mathrm{Hz}}$ and the loss factors $\eta$ (representing damping) correspond to the real part and to the ratio of imaginary and real part of the complex eigen-frequencies

$$
\begin{gathered}
f_{\mathrm{Hz}}=\frac{\sqrt{\operatorname{Re}\left[\omega^{2}\right]}}{2 \pi}, \\
\eta=\frac{\operatorname{Im}\left[\omega^{2}\right]}{\operatorname{Re}\left[\omega^{2}\right]} .
\end{gathered}
$$

This numerical solution converges for a small stopping tolerance to the exact solution. That is why this method is taken as the reference one, when assessing the accuracy of the simplified methods considered next.

\subsection{Dynamic effective thickness (DET)}

To our best knowledge, only one effective thickness approach for modal analysis of laminated glass beams can be found in literature. This approach by LópezAenlle and Pelayo [6] is derived from a closed-form solution of free vibration for a three-layer beam with simply-supported ends. For the other boundary conditions, the authors adjust the formulation using the wavenumbers of an Euler-Bernoulli beam, see ahead Eq. (7) and Table 2

The analytical expression for this dynamic effective thickness has the following form

$$
h_{\mathrm{ef}}(\omega)=\sqrt[3]{\left(h_{1}^{3}+h_{3}^{3}\right)\left(1+\frac{Y}{1+\frac{h_{1}}{q(\omega)\left(h_{1}+h_{3}\right)}}\right)},
$$

where $Y$ is a geometric parameter, which depends only on the thicknesses of layers and is given by

$$
Y=\frac{12 h_{1} h_{3}\left(0.5 h_{1}+h_{2}+0.5 h_{3}\right)}{\left(h_{1}^{3}+h_{3}^{3}\right)\left(h_{1}+h_{3}\right)},
$$

and $q(\omega)$ is a material parameter given by the identity

$$
q(\omega)=\frac{G_{2}(\omega)}{E_{1} h_{3} h_{2} \beta_{n}^{2}} .
$$

In this equation, $G_{2}(\omega)$ is the frequency-dependent complex shear modulus of the interlayer, $E_{1}$ is the Young modulus of glass layers, and $\beta_{n}$ is the wavenumber corresponding to the given boundary conditions and to the $n$-th mode, see ahead Table 2 Because the shear modulus $G_{2}(\omega)$ is complex-valued, the effective thickness from Eq. (5) is also complex.

Then, the complex-valued natural angular frequencies can be expressed analytically, similarly as for the monolithic beam

$$
\omega^{2}=\frac{\beta_{n}^{4} E_{1} h_{\mathrm{ef}}^{3}(\omega)}{12 \bar{m}},
$$



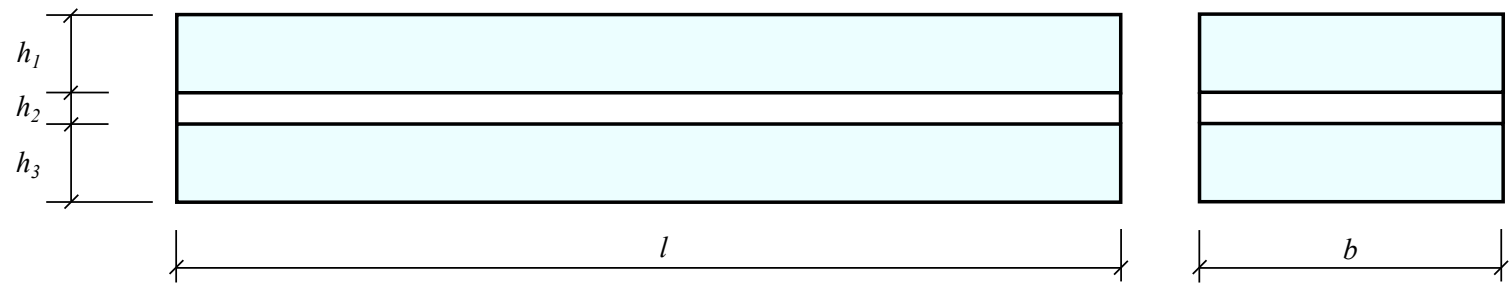

Figure 2. The layout of a three-layered laminated glass beam.

where

$$
\bar{m}=\rho_{1} h_{1}+\rho_{2} h_{2}+\rho_{3} h_{3}
$$

is the effective mass per unit of length and width. The natural frequencies $f_{\mathrm{Hz}}$ and loss factors $\eta$ are obtained from this complex-valued angular frequency $\omega$ according to Eq. (4).

\subsection{Enhanced EFFECTIVE THiCKness (EET)}

Firstly, we introduce the formula for the deflectioneffective thickness according to Galuppi and RoyerCarfagni [7, and subsequently, we propose its extension to the free vibration analysis.

To shorten the notation, $H$ is defined as a distance of centerlines of glass layers, so that

$$
H=h_{2}+0.5\left(h_{1}+h_{3}\right) .
$$

Further, $I_{t o t}$ is the second moment of area of glass layers for the monolithic case

$$
I_{\text {tot }}=I_{1}+I_{3}+\frac{A_{1} A_{3}}{A_{1}+A_{3}} H^{2},
$$

and $I_{s}$ is the last term from 11 divided by the width of the beam $b$, thus

$$
I_{s}=\frac{h_{1} h_{3}}{h_{1}+h_{3}} H^{2} .
$$

Using these quantities, the enhanced effective thickness for the deflection under static loading from [7] has the following form

$$
h_{\mathrm{ef}}=\sqrt[3]{\frac{1}{\frac{\zeta}{h_{1}^{3}+h_{3}^{3}+12 I_{s}}+\frac{1-\zeta}{h_{1}^{3}+h_{3}^{3}}}},
$$

where $\zeta$ is the coefficient of shear cohesion

$$
\zeta=\frac{1}{1+\frac{I_{1}+I_{3}}{\mu I_{\text {tot }}} \frac{A_{1} A_{3}}{A_{1}+A_{3}} \psi},
$$

and $\mu$ is the non-dimensional ratio of the glass and interlayer stiffnesses

$$
\mu=\frac{G_{2} b}{E_{1} h_{2}} .
$$

Finally, $\psi$ is a coefficient which depends on the shape of the deflection curve and is described by the following equation

$$
\psi=\frac{\int_{0}^{l}\left(g^{\prime \prime}(x)\right)^{2} \mathrm{~d} x}{\int_{0}^{l}\left(g^{\prime}(x)\right)^{2} \mathrm{~d} x},
$$

\begin{tabular}{lr}
\hline Geometry & \\
\hline length & $l=1 \mathrm{~m}$ \\
width & $b=0.1 \mathrm{~m}$ \\
thicknesses of glass & $h_{1}=h_{3}=10 \mathrm{~mm}$ \\
thickness of interlayer & $h_{2}=1.52 \mathrm{~mm}$ \\
\hline Glass & \\
\hline density, ratio & $\rho_{1}=\rho_{3}=2500 \mathrm{~kg} / \mathrm{m}^{3}$ \\
Poisson's & $\nu_{1}=\nu_{3}=0.22$ \\
Young's modulus & $E_{1}=E_{3}=72 \mathrm{GPa}$ \\
\hline Interlayer & \\
\hline density & \\
Poisson's ratio & $\rho_{2}=1100 \mathrm{~kg} / \mathrm{m}^{3}$ \\
Prony series for GMM & $\nu_{2}=0.49$ \\
\hline
\end{tabular}

TABLE 1. The properties of laminated glass beams (GMM - Generalized Maxwell Model).

where the deflection curve is represented by the function $g(x)$, which describes the shape of the curve regardless of the stiffness of the beam.

This deflection-effective thickness was derived by minimization of the strain energy functional for a laminated glass beam in bending under static loading. For its use in modal analysis, we made just two intuitive adjustments of this method:

(1.) We used the complex-valued interlayer shear modulus $G_{2}(\omega)$ in Eq. 15 .

(2.) We replaced the shape function of the deflection under static loading in Eq. (16) with the one corresponding to the $n$-th mode shape of a monolithic beam under given boundary conditions.

This adjustment leads to a complex effective thickness in Eq. 13, similarly to the DET approach. For the evaluation of the natural frequencies and the damping, we again use Eq. (4).

\section{Comparison OF Methods}

\subsection{EXAMPLES}

In this section, we discuss the applicability of effective thickness approaches in modal analysis and how our adjustment changes the errors of the method. The comparison is provided for three types of boundary conditions: simply-supported, fixed-fixed, and free-free. 


\begin{tabular}{lr}
\hline \multicolumn{1}{c}{ Mode shape functions } \\
\hline $\begin{array}{l}\text { simply-supported beam } \\
\text { fixed-fixed beam }\end{array}$ \\
$\qquad(x)=\sin \frac{n \pi x}{l}$ \\
free-free beam \\
$\qquad g(x)=\sinh \beta_{n} x+\sin \beta_{n} x-\frac{\sinh \beta_{n} l-\sin \beta_{n} l}{\cosh \beta_{n} l-\cos \beta_{n} l}\left(\cosh \beta_{n} x+\cos \beta_{n} x\right)$ \\
\hline
\end{tabular}

TABlE 2. The overview of the $n$-th mode shape functions for the coordinate $x \in\langle 0, l\rangle$ for a simply-supported, fixed-fixed, and free-free beam. (The products of the wavenumbers and the beam length $\beta_{n} l$ for the first three modes are $\beta_{1} l=4.7300, \beta_{2} l=7.8532$, and $\beta_{3} l=10.996$.)

The geometry and the material properties of glass and interlayer appear in Table 1 . Whereas glass is treated as an elastic material, the viscoelastic behavior of polymer, which is in our case polyvinyl butyral (PVB), is described using a generalized Maxwell model, whose parameters are taken from [3. Due to the temperature-sensitiveness of PVB, two different ambient temperatures were assumed - the room temperature $25^{\circ} \mathrm{C}$ and the temperature $50^{\circ} \mathrm{C}$ corresponding to an external panel under summer sunlight.

\subsection{Evaluation of Shape COEFFicients}

Recall that our extension of the enhanced effective thickness approach [7] to the modal analysis of laminated glass beams requires the evaluation of the shape coefficients $\psi$ according Eq. 16). For these coefficients, we need mode shape functions corresponding to the three given boundary conditions. Their overview taken from [11] is shown in Table 2, For a simplysupported beam, one closed-form expression can be written for all mode shape functions; however, the formulas for the other boundary conditions are more involved and contain a product of the wavenumbers $\beta_{n}$ and the beam length $l$ that needs to be evaluated numerically from a characteristic equation for free vibrations 11 .

Then, we used the integral formula Eq. (16) and evaluated, for all boundary conditions, the shape coefficients $\psi$ summarized in Table 3 using the mode shape functions for a monolithic Euler-Bernoulli beam from Table 2 .

With these results at hand, we can calculate the complex enhanced effective thickness and subsequently the natural frequencies and the loss factors for our comparison.

\subsection{Results AND DisCUSSION}

In this section, the results obtained by the reference method (RM), the effective thickness approaches from [6] (DET) or from [7] (EET), their errors, and their comparison are shown. The first three natural frequencies $f_{\mathrm{Hz}}$ and loss factors $\eta$ are summarized in Table 4. The errors of the effective thickness approaches are evaluated against the reference method based on the finite element complex-eigenvalue solver.

\begin{tabular}{lccc}
\hline \multicolumn{4}{c}{ Shape coefficients $\psi$} \\
\hline beam & 1 & 2 & 3 \\
\hline simply-supported & $\frac{\pi^{2}}{l^{2}}$ & $\frac{(2 \pi)^{2}}{l^{2}}$ & $\frac{(3 \pi)^{2}}{l^{2}}$ \\
fixed-fixed & $\frac{40.7}{l^{2}}$ & $\frac{82.6}{l^{2}}$ & $\frac{148}{l^{2}}$ \\
free-free & $\frac{10.1}{l^{2}}$ & $\frac{34.9}{l^{2}}$ & $\frac{78.2}{l^{2}}$ \\
\hline
\end{tabular}

TABLE 3. Summary of the shape coefficients $\psi$ for three different boundary conditions and the first three mode shapes.

It is evident from the first two subtables in Table 4 that the adjusted EET approach gives the same results as the DET method for the simply-supported beam for both temperatures. Both effective thickness methods give the errors in natural frequencies less than $1 \%$ and in loss factors less than $10 \%$. For the room temperature, even the errors in loss factors are under $1 \%$.

For the two other boundary condition, the DET approach provides quite good predictions for natural frequencies with the errors under $13 \%$. However, the errors in loss factors can be very high (up to 80\%). Therefore, this method is mostly unable to provide a good estimation of damping.

The adjusted EET approach helps to overcome these inaccuracies. For the fixed-fixed beam, this approach gives the largest error in natural frequencies $3 \%$ (against the $8 \%$ error by the DET) and in loss factors $9 \%$ (against the $44 \%$ error by the DET). For the free-free beam, the largest value of errors in natural frequencies is $3 \%$ (against the $13 \%$ error by the DET) and in loss factors $19 \%$ (against the $78 \%$ error by the DET).

This demonstrates that our intuitive extension of the variationally-based enhanced effective thickness concept from [7] provides better (or for the simplysupported beam the same) estimates of both - the natural frequencies and loss factors for laminated glass beams. 


\begin{tabular}{|c|c|c|c|c|c|c|c|c|c|c|c|}
\hline \multicolumn{12}{|c|}{ Simply-supported beam at $25^{\circ} \mathrm{C}$} \\
\hline \multirow[t]{2}{*}{ Mode } & \multicolumn{3}{|c|}{ Natural frequency $[\mathrm{Hz}]$} & \multicolumn{2}{|c|}{ Error $[\%]$} & \multirow[t]{2}{*}{ Mode } & \multicolumn{3}{|c|}{ Loss factor $[\%]$} & \multicolumn{2}{|c|}{ Error [\%] } \\
\hline & $\mathrm{RM}$ & $\mathrm{DET}$ & EET & DET & EET & & $\mathrm{RM}$ & DET & EET & DET & EET \\
\hline 1 & 52.03 & 52.07 & 52.07 & 0.09 & 0.09 & 1 & 1.93 & 1.93 & 1.93 & -0.25 & -0.25 \\
\hline 2 & 197.9 & 198.5 & 198.5 & 0.30 & 0.30 & 2 & 4.24 & 4.23 & 4.23 & -0.32 & -0.32 \\
\hline 3 & 419.1 & 421.4 & 421.4 & 0.56 & 0.56 & 3 & 6.15 & 6.14 & 6.14 & -0.20 & -0.20 \\
\hline \multicolumn{12}{|c|}{ Simply-supported beam at $50^{\circ} \mathrm{C}$} \\
\hline \multirow[t]{2}{*}{ Mode } & \multicolumn{3}{|c|}{ Natural frequency $[\mathrm{Hz}]$} & \multicolumn{2}{|c|}{ Error $[\%]$} & Mode & \multicolumn{3}{|c|}{ Loss factor $[\%]$} & \multicolumn{2}{|c|}{ Error $[\%]$} \\
\hline & $\mathrm{RM}$ & DET & EET & $\mathrm{DET}$ & EET & & $\mathrm{RM}$ & $\mathrm{DET}$ & EET & DET & EET \\
\hline 1 & 30.81 & 30.97 & 30.97 & 0.53 & 0.53 & 1 & 24.41 & 22.17 & 22.17 & -9.18 & -9.18 \\
\hline 2 & 108.1 & 108.5 & 108.5 & 0.42 & 0.42 & 2 & 16.89 & 16.25 & 16.25 & -3.81 & -3.81 \\
\hline 3 & 232.0 & 233.1 & 233.1 & 0.47 & 0.47 & 3 & 14.46 & 13.90 & 13.90 & -3.84 & -3.84 \\
\hline \multicolumn{12}{|c|}{ Fixed-fixed beam at $25^{\circ} \mathrm{C}$} \\
\hline \multirow[t]{2}{*}{ Mode } & \multicolumn{3}{|c|}{ Natural frequency $[\mathrm{Hz}]$} & \multicolumn{2}{|c|}{ Error $[\%]$} & \multirow[t]{2}{*}{ Mode } & \multicolumn{3}{|c|}{ Loss factor $[\%]$} & \multicolumn{2}{|c|}{ Error [\%] } \\
\hline & $\mathrm{RM}$ & $\mathrm{DET}$ & EET & $\mathrm{DET}$ & EET & & $\mathrm{RM}$ & $\mathrm{DET}$ & EET & DET & EET \\
\hline 1 & 110.2 & 115.4 & 111.3 & 4.78 & 1.09 & 1 & 5.42 & 3.09 & 5.08 & -43.05 & -6.27 \\
\hline 2 & 285.3 & 301.4 & 292.7 & 5.65 & 2.59 & 2 & 7.15 & 5.29 & 6.50 & -25.94 & -9.02 \\
\hline 3 & 527.2 & 556.3 & 541.1 & 5.53 & 2.65 & 3 & 7.86 & 6.74 & 7.56 & -14.28 & -3.82 \\
\hline \multicolumn{12}{|c|}{ Fixed-fixed beam at $50^{\circ} \mathrm{C}$} \\
\hline \multirow[t]{2}{*}{ Mode } & \multicolumn{3}{|c|}{ Natural frequency $[\mathrm{Hz}]$} & \multicolumn{2}{|c|}{ Error $[\%]$} & Mode & \multicolumn{3}{|c|}{ Loss factor $[\%]$} & Err & {$[\%]$} \\
\hline & $\mathrm{RM}$ & $\mathrm{DET}$ & EET & DET & EET & & $\mathrm{RM}$ & $\mathrm{DET}$ & EET & DET & EET \\
\hline 1 & 60.06 & 64.63 & 60.21 & 7.62 & 0.25 & 1 & 12.99 & 18.74 & 12.72 & 44.19 & -2.14 \\
\hline 2 & 160.2 & 164.6 & 160.9 & 2.79 & 0.46 & 2 & 11.51 & 14.91 & 11.80 & 29.52 & 2.49 \\
\hline 3 & 308.8 & 314.0 & 310.2 & 1.66 & 0.43 & 3 & 10.98 & 12.89 & 10.87 & 17.39 & -1.03 \\
\hline & & & & Free- & ree bc & am at 2 & $5^{\circ} \mathrm{C}$ & & & & \\
\hline Mode & Natur & 1 freque & cy $[\mathrm{Hz}]$ & Erro & {$[\%]$} & Mode & Los & factor & & Err & {$[\%]$} \\
\hline & $\mathrm{RM}$ & $\mathrm{DET}$ & EET & $\mathrm{DET}$ & EET & & $\mathrm{RM}$ & $\mathrm{DET}$ & EET & $\mathrm{DET}$ & EET \\
\hline 1 & 117.8 & 115.4 & 118.4 & -2.00 & 0.56 & 1 & 1.73 & 3.09 & 1.50 & 78.05 & -13.64 \\
\hline 2 & 307.9 & 301.4 & 314.1 & -2.12 & 2.00 & 2 & 4.13 & 5.29 & 3.37 & 28.20 & -18.41 \\
\hline 3 & 568.4 & 556.3 & 584.5 & -2.12 & 2.84 & 3 & 5.77 & 6.74 & 5.04 & 16.74 & -12.64 \\
\hline & & & & Free- & ree bc & am at 5 & $0^{\circ} \mathrm{C}$ & & & & \\
\hline Mode & Naturs & freque & cy $[\mathrm{Hz}]$ & Erro & {$[\%]$} & Mode & Los & factor & & Err & {$[\%]$} \\
\hline & $\mathrm{RM}$ & DET & EET & DET & EET & & $\mathrm{RM}$ & DET & EET & DET & EET \\
\hline 1 & 73.95 & 64.63 & 74.62 & -12.60 & 0.90 & 1 & 26.44 & 18.74 & 25.39 & -29.13 & -3.96 \\
\hline 2 & 170.5 & 164.6 & 175.6 & -3.45 & 2.97 & 2 & 20.85 & 14.91 & 22.20 & -28.51 & 6.48 \\
\hline 3 & 322.5 & 314.0 & 325.3 & -2.66 & 0.86 & 3 & 17.57 & 12.89 & 18.20 & -26.61 & 3.62 \\
\hline
\end{tabular}

TABLE 4. Natural frequencies and loss factors for the three-layer laminated glass beam for the first three modes determined by the reference method (RM), the dynamic effective thickness method (DET), and the adjusted enhanced effective thickness approach (EET) with their errors against the reference method (RM). 


\section{Conclusions}

Finally, we would like to summarize that in this paper

- we verified the effective thickness approaches against the finite element complex-eigenvalue solver, and

- we proposed a new dynamic effective thickness concept derived from [7].

It follows from our comparison that this extended enhanced effective thickness method

- provides the same results for the simply-supported beam as the dynamic effective thickness from 6] with the errors less than $1 \%$ in natural frequencies and $10 \%$ in loss factors, and

- improves the estimates of natural frequencies and loss factors for the other two boundary conditions with the errors less than $3 \%$ in natural frequencies and $19 \%$ in loss factors.

In our future work, we would like to justify this intuitive approach by rigorously extending the variationally-based derivation of the effective thickness from [7] to the dynamic problems.

\section{ACKNOWLEDGEMENTS}

This publication was supported by the Czech Science Foundation, the grant No. 16-14770S and by the Grant Agency of the Czech Technical University in Prague, grant No. SGS17/043/OHK1/1T/11.

\section{REFERENCES}

[1] M. Haldimann, A. Luible, M. Overend. Structural Use of Glass, vol. 10 of Structural Engineering Documents. IABSE, Zürich, Switzerland, 2008.

[2] Z. Huang, Z. Qin, F. Chu. Damping mechanism of elastic-viscoelastic-elastic sandwich structures. Composite Structures 153:96-107, 2016. DOI:10.1016/j.compstruct.2016.05.105
[3] Y. Shitanoki, S. Bennison, Y. Koike. A practical, nondestructive method to determine the shear relaxation modulus behavior of polymeric interlayers for laminated glass. Polymer Testing 37:59-67, 2014. DOI:10.1016/j.polymertesting.2014.04.011

[4] I. Mohagheghian, Y. Wang, L. Jiang, et al. Quasi-static bending and low velocity impact performance of monolithic and laminated glass windows employing chemically strengthened glass. European Journal of Mechanics - A/Solids 63:165-186, 2017. DOI:10.1016/j.euromechsol.2017.01.006.

[5] L. Andreozzi, S. B. Bati, M. Fagone, et al. Dynamic torsion tests to characterize the thermo-viscoelastic properties of polymeric interlayers for laminated glass. Construction and Building Materials 65:1-13, 2014. DOI:10.1016/j.conbuildmat.2014.04.003

[6] M. López-Aenlle, F. Pelayo. Dynamic effective thickness in laminated-glass beams and plates. Composites: Part B 67:332-347, 2014.

[7] L. Galuppi, G. F. Royer-Carfagni. Effective thickness of laminated glass beams: New expression via a variational approach. Engineering Structures 38:53 - 67, 2012. DOI:10.1016/j.engstruct.2011.12.039.

[8] L. Galuppi, G. Royer-Carfagni. The effective thickness of laminated glass: Inconsistency of the formulation in a proposal of EN-standards. Composites Part B: Engineering 55:109-118, 2013.

[9] F. Pelayo, M. López-Aenlle. Natural frequencies and damping ratios of multi-layered laminated glass beams using a dynamic effective thickness. Journal of Sandwich Structures and Materials 0:1-25, 2017. DOI:10.1177/1099636217695479

[10] K. Schreiber. Nonlinear Eigenvalue Problems: Newton-type Methods and Nonlinear Rayleigh Functionals. Ph.D. thesis, Technischen Universitat Berlin, 2008.

[11] R. W. Clough, J. Penzien. Dynamics of structures. Computers \& Structures, Inc, 2003. 